\title{
A NONDESTRUCTIVE PRESCREENING METHOD FOR BONE COLLAGEN CONTENT USING MICRO-COMPUTED TOMOGRAPHY
}

\author{
Jennifer A Tripp ${ }^{1,2} \cdot$ Maria E Squire $^{3} \cdot$ Julie Hamilton $^{4} \cdot$ Robert E M Hedges $^{4}$
}

ABSTRACT. Isolation of bone collagen for radiocarbon dating is a labor-intensive and time-consuming process that sometimes results in unacceptably low protein recovery. In preliminary studies reported here, micro-computed tomography (microCT), a nondestructive technique that uses X-rays to produce high-resolution three-dimensional images of mineralized materials such as bone, offers promise as a suitable prescreening option for bones of questionable preservation. We have found that the bone volume fraction calculated by the scanner software correlates well with collagen recovery in 4 analyzed bones from Etton, United Kingdom.

\section{INTRODUCTION}

Bone undergoes a number of morphological changes upon decay and diagenesis, including changes in crystallinity, porosity, and histology (Hedges 2002). Radiocarbon dating, stable isotope analysis, and extraction of ancient DNA all depend upon the preservation of organic matter within a bone, a parameter difficult to discern prior to extraction of the desired protein or nucleic acid. The organic preservation depends upon many factors, including immediate post mortem treatment (i.e. cooking or defleshing), temperature and hydration of the burial site, and time since death (Collins et al. 2002). Isolation of bone collagen for ${ }^{14} \mathrm{C}$ dating is a labor-intensive and time-consuming process that sometimes results in unacceptably low protein recovery (Bronk Ramsey et al. 2004), so a method to predetermine protein preservation would be useful.

Microstructural features of a bone, especially porosity, have been found to correlate with protein content as measured by \% nitrogen within the bone (Nielsen-Marsh and Hedges 1999, 2000; TurnerWalker and Syversen 2002). Thus, examination of a bone's microstructure and its correlation with protein content should, in theory, enable the development of a prescreening method to predict collagen recovery, allowing for bones with low collagen content to be excluded before the costly pretreatment process begins. Unfortunately, the 2 most common methods for measuring bone porosity, water vapor adsorption and mercury intrusion porosimetry ( $\mathrm{HgIP})$, are time-consuming and destructive, requiring at least $100 \mathrm{mg}$ of sample for adsorption (Hedges et al. 1995) and $300 \mathrm{mg}$ for HgIP (Nielsen-Marsh and Hedges 1999). For rare or valuable samples, destruction of this much material is impracticable, and in addition there is no guarantee that the sample taken for pore size measurements would be chemically and morphologically identical to that later taken for dating, even if they are from the same bone.

We have therefore sought development of a technique that could be used to study the preservation of bones and offer a measurement that correlates with bone preservation, particularly with collagen recovery. Ideally, a rapid, nondestructive method that provides information about a large volume of bone would offer the most promise as a prescreening method, both to choose the best bone from an assemblage as well as the best location from within a bone to sample for dating. Micro-computed

\footnotetext{
${ }^{1}$ Department of Chemistry, University of Scranton, 800 Linden Street, Scranton, Pennsylvania 18510, USA.

${ }^{2}$ San Francisco State University, Department of Chemistry \& Biochemistry, 1600 Holloway Avenue, San Francisco, California 94132, USA. Email: tripp@sfsu.edu.

${ }^{3}$ Department of Biology, University of Scranton, 800 Linden Street, Scranton, Pennsylvania 18510, USA.

${ }^{4}$ Research Laboratory for Archaeology and the History of Art, Dyson Perrins Building, South Parks Road, University of Oxford, Oxford, OX1 3QY, United Kingdom.
}

C 2010 by the Arizona Board of Regents on behalf of the University of Arizona Proceedings of the 20th International Radiocarbon Conference, edited by A J T Jull RADIOCARBON, Vol 52, Nr 2-3, 2010, p 612-619 
tomography (microCT) promises to be such a technique, and here we describe our preliminary results from the analysis of archaeological bones using this instrument.

MicroCT is a state-of-the-art technique for the nondestructive analysis of mineralized objects such as bone (Rüegsegger et al. 1996). Images are obtained using a cone-shaped beam X-ray that is projected toward the specimen, a charge-coupled device (CCD) array detector that captures the images, and a scintillator that converts $\mathrm{X}$-rays into visible rays. This creates stacks of sequential two-dimensional cross-sectional images of the specimen, which are then reconstructed to generate a high-resolution three-dimensional image of the object (Rüegsegger et al. 1996; Müller et al. 1998). The resolution of the image can be as high as 5-6 $\mu \mathrm{m}$, depending on the instrument and the size of the specimen. Using image analysis software, standard morphometric indices of bone (Parfitt et al. 1987) including bone volume, tissue volume, bone surface, bone volume fraction, connectivity density, trabecular thickness, trabecular number, and trabecular separation, can be determined. While the indices described are for trabecular bone, they can be applied to images from any object, including cortical bone (Laib et al. 2000; Squire et al. 2008), teeth (Peters et al. 2000), or blood vessels (Brown et al. 2005), for which indices such as volume, surface area, and thickness, are desired. One important benefit of nondestructive imaging of the specimens is that additional assays on the same specimen, such as mechanical properties testing or chemical composition including ${ }^{14} \mathrm{C}$, are possible after scanning. In addition, imaging and analysis of specimens that are too valuable to destroy, such as fossils or museum artifacts, is possible.

While computed tomography (CT) has been used to study archaeological faunal bones (Lam et al. 1998), the Iceman (Murphy et al. 2003), and the famous Queen Nefertiti statue (Huppertz et al. 2009), amongst other artifacts, and microCT has been used to study fossil teeth (Olejniczak and Grine 2006), to our knowledge this is the first time that microCT has been applied to archaeological bones in a study of protein preservation. Here, we present the preliminary results from our ongoing study and discuss the potential of microCT for studies of bone preservation and as a prescreening method for collagen content.

\section{EXPERIMENTAL}

\section{Samples}

A number of sheep and cattle bones from the site of Etton, located near Peterborough in Cambridgeshire, England, were obtained. This site has variable bone preservation, with some excavated bones having very high collagen recovery, and others containing no collagen at all. Because samples for ${ }^{14} \mathrm{C}$ dates are typically taken from the diaphyses of long bones, we have chosen to analyze cortical bone samples. Sample information for the 4 bones used in our preliminary study is listed in Table 1.

Table 1 Sample information for bones used in this study (Brock et al. 2007).

\begin{tabular}{lllclll}
\hline Sample $\mathrm{nr}$ & Species & Element & \% collagen & $\mathrm{C} / \mathrm{N}$ & $\delta^{13} \mathrm{C}$ & $\delta^{15} \mathrm{~N}$ \\
\hline ET12 & cattle & tibia & 5.26 & 3.34 & -21.72 & 4.58 \\
ET18 & cattle & tibia & 0.03 & 9.12 & - & - \\
ET46 & sheep & humerus & 13.15 & 3.35 & -22.24 & 6.62 \\
ET50 & sheep & humerus & 0.00 & - & - & - \\
\hline
\end{tabular}




\section{Instrument}

Bone samples were scanned at the University of Scranton using a MicroCT 80 scanning system $(\mu \mathrm{CT} 80$, Scanco Medical, Switzerland). All samples were scanned vertically in a cylindrical sample holder $(60 \mathrm{~mm} \mathrm{D} \times 85 \mathrm{~mm} \mathrm{H})$, at a voltage of $55 \mathrm{kV}$ and intensity of $45 \mu \mathrm{A}$, and at 2 different resolutions: $60 \mu \mathrm{m}$ (isotropic voxel size) and $30 \mu \mathrm{m}$ (isotropic voxel size). The time required to complete a scan of a $48-\mathrm{mm}$ specimen is $4 \mathrm{hr}$ at the $60-\mu \mathrm{m}$ resolution and $15 \mathrm{hr}$ at the $30-\mu \mathrm{m}$ resolution.

\section{Image Analysis}

Bone volume fraction (BV/TV) was analyzed on a 9-mm region of each bone specimen. The chosen volume of interest (VOI) was in close proximity to the area from which a sample was taken for isolation of collagen and stable isotope analysis for the prior study (Brock et al. 2007), when that could be determined. Two types of contour lines were drawn: (1) a contour along the periosteal surface of the bone coupled to a second around the endosteal surface of the bone; and (2) a series of cylinders drawn within the bone structure. Care was taken to exclude from analysis any rocks or other debris present in the diaphyseal shaft and, for the cylinders, any cracks or large pores within the bone structure. Prior to evaluation, a Gaussian filter (sigma $=0.8$ voxels) removed noise from the images and segmentation, which separated bone from background, was done at thresholds ranging from 250 to 600 (in increments of 50). The VOI was evaluated by the Scanco software at each threshold, and the bone volume fraction values were recorded.

\section{RESULTS \& DISCUSSION}

This paper describes the analysis of 4 bones of variable preservation from the site of Etton, UK (Table 1). We are in the process of scanning and analyzing over 20 samples from this site, and anticipate that with further work the patterns seen here will be reproduced and reinforced.

\section{Images}

The images themselves show clear visual differences between the bones with high collagen recovery and those with little or no collagen present (Figures 1 and 2). The fabric of the well-preserved bones in general appears more uniform, while the bones that contain little collagen appear more variable and porous. This is what we would expect from previous studies of diagenetic alteration of bone, which found that diagenesis leads to an increase in the porosity of bone (Nielsen-Marsh and Hedges 1999; Hedges 2002).

\section{Image Analysis}

The search for a quantitative microCT measurement for diagenetic change led us to the use of bone volume fraction (BV/TV). Bone volume (BV) and total volume (TV) of the sample can be computed from the microCT software, and the ratio of these values (BV/TV) offers a measure of the amount of bone tissue present within a given volume of bone sample; this ratio represents, in other words, the percent of the bone that is composed of bone mineral as opposed to non-mineralized tissue or empty space.

Thus, the BV/TV can be interpreted as a measure of the percent of the total volume that contains mineralized tissue; the closer this ratio is to 1 , the fewer pores are present within the bone. The BV/ $\mathrm{TV}$ is calculated by the scanner software using a sample volume designated by the user. For modern samples of cortical bone, the volume is normally determined by drawing contour lines around the periosteal and endosteal surfaces of successive images. For archaeological bones with good struc- 

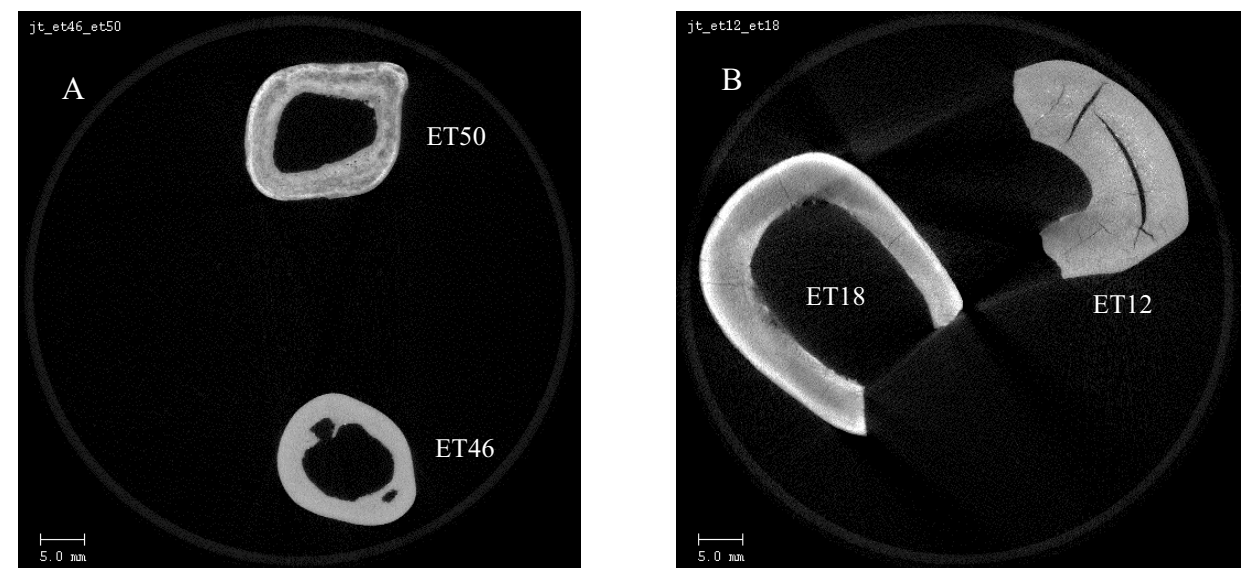

Figure 1 MicroCT images of bones used in this study: (A) ET46 and ET50 (sheep humeri); (B) ET12 and ET18 (cattle tibiae).
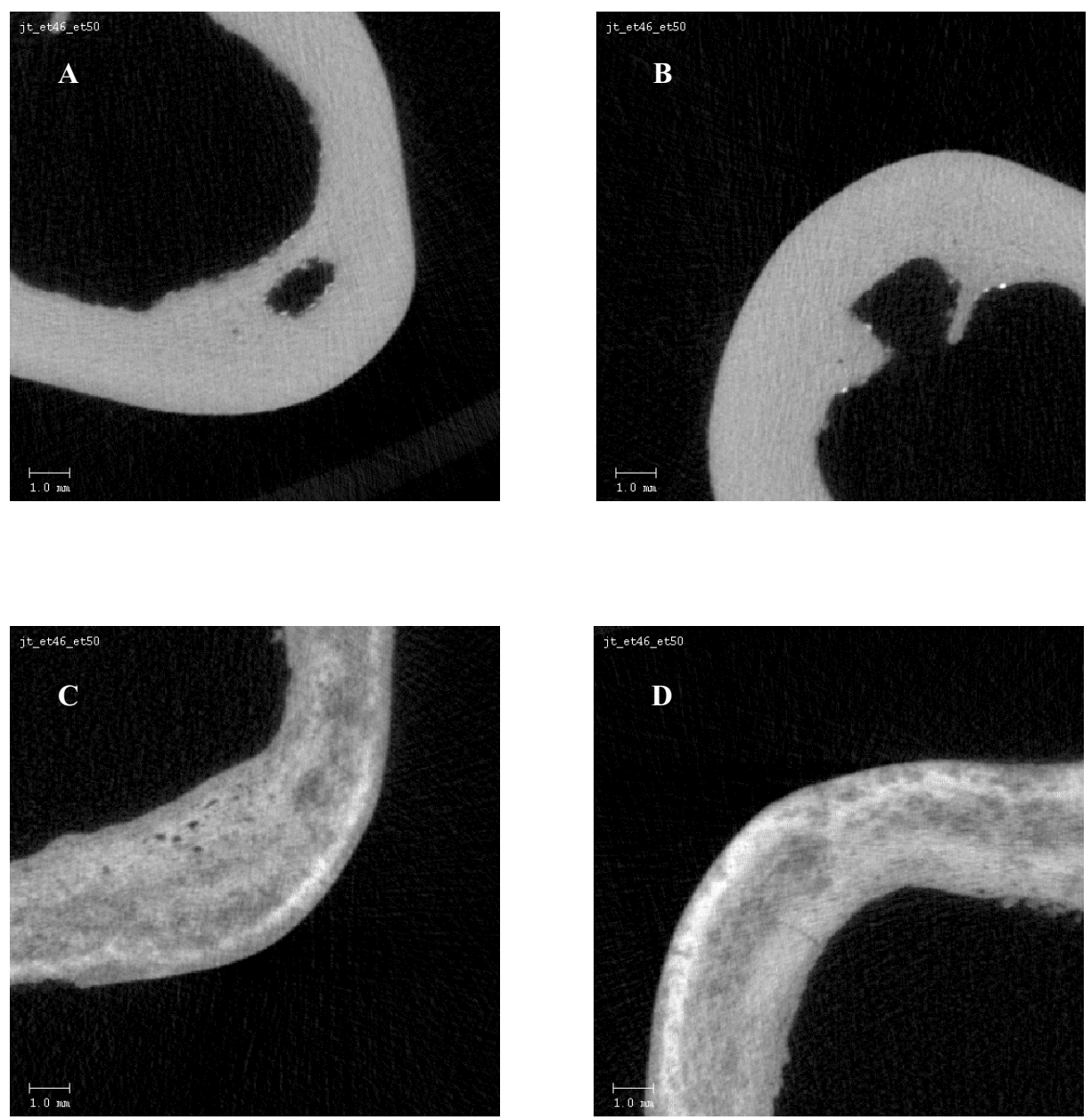

Figure 2 Expanded microCT images of 2 of the bones used in this study: (A) and (B) show ET46, which had $13.15 \%$ collagen recovery; (C) and (D) show ET50, from which no collagen could be recovered. 
tural integrity, this method would also work well, but for bones that are particularly dirty, cracked, or fragmented, such as most of those used in this study, the opportunity to introduce foreign substances of higher density (i.e. sediment particles or small rocks), or cracks (which would artificially lower the overall $\mathrm{BV} / \mathrm{TV}$ of the bone) into the analyzed volume is great.

Thus, for the Etton bones we explored a method in which a number of cylindrical volumes are designated within each bone and averaged together. The cylinders can be placed within the bone in order to minimize the inclusion of foreign particles or large cracks, and fragmented bones can have one or more cylinders of identical volume placed within each section so that a BV/TV value can be calculated for the bone by a simple average rather than a more complicated calculation involving a weighted average based upon the volume of each fragment. We drew 4 cylindrical volumes within each bone (except for ET12 for which 3 cylinders were designated) and averaged the values together.

An additional important parameter for the determination of BV/TV using microCT is the threshold, a value the user inputs that enables the computer to distinguish bone from background. For our samples, there was not an obvious value to choose, so we studied a range of threshold values from 250600 in order to determine the best value to use for our bone volume fraction measurements. The threshold is a grayscale pixel value that binarizes the images to black and white. At low threshold values, more of the volume is designated as bone and calculated BV/TV values are at or close to one. As the threshold is increased, the BV/TV decreases, and we found that the rate of decrease differed based on bone preservation (Figure 3). As expected, BV/TV decreases with increasing threshold, but the better preserved bones (ET12 and ET46) maintain a high BV/TV at higher thresholds compared to the poorly preserved bones (ET18 and ET50). The difference between bones with high collagen recovery and those with very low collagen recovery is particularly apparent at a threshold of 500 .

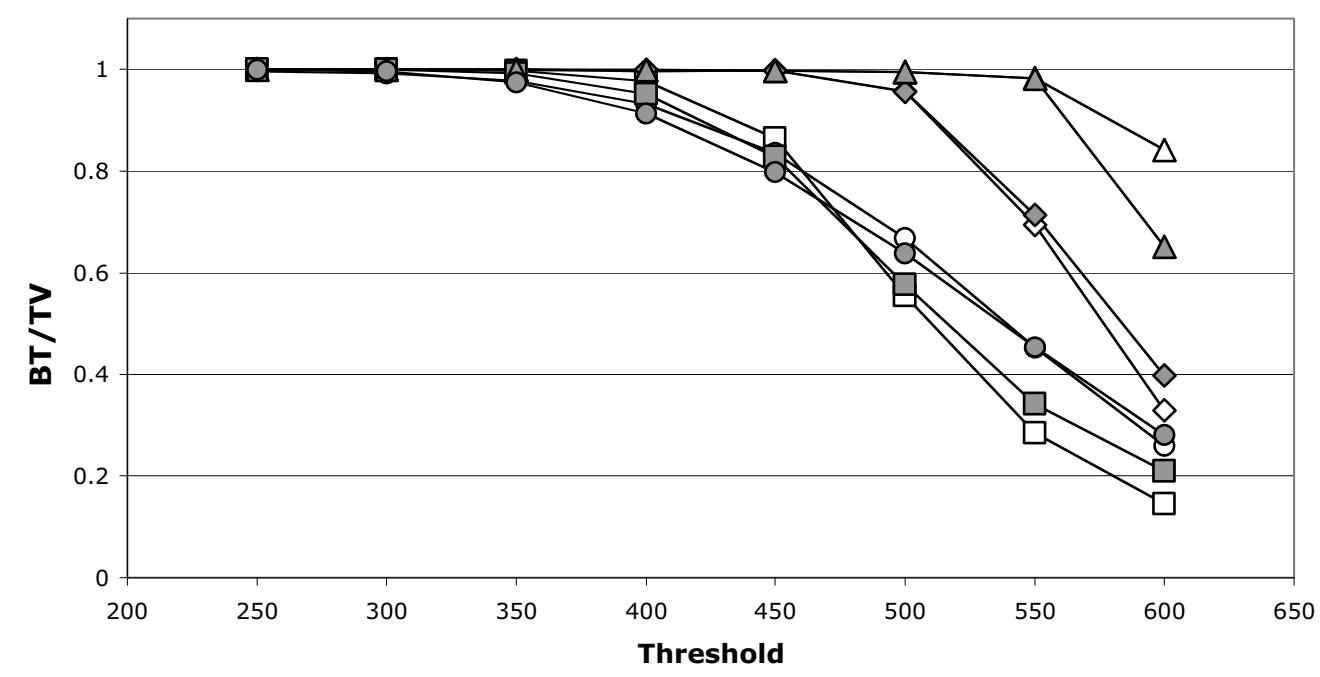

Figure $3 \mathrm{BV} / \mathrm{TV}$ plotted against threshold for the 4 Etton bones analyzed. The cylindrical analysis method was used and each point is the average value for 4 cylindrical contours drawn within the bone (3 for ET12). $\diamond=$ ET12, $\square=\mathrm{ET} 18, \triangle=\mathrm{ET} 46, \mathrm{O}=\mathrm{ET} 50$. Shaded points are data calculated from high-resolution images and white points from low-resolution images. 
We initially collected both high- and low-resolution images and analyzed them using the cylindrical method for all 4 bones. Figure 3 demonstrates that very close results at every threshold are obtained for BV/TV with high- and low-resolution images. This implies that low-resolution scans are sufficient for the determination of BV/TV for the archaeological bones we have examined. High-resolution scans take approximately 4 times longer than low-resolution scans, and because they contain more slices per unit volume, the analysis of the high-resolution images also takes longer. From an economic standpoint, taking into account the time spent by laboratory personnel using microCT, the use of low-resolution images to determine bone preservation is preferred.

In order to examine the utility of the analytical method using cylindrical contours, we used the same slice range of the same images as above to calculate BV/TV for 2 of the bones using contours drawn around the whole bone. ET46 and ET50 are both intact bones with good macrostructural integrity and inclusion of very little foreign material in the diaphyseal shaft, so they were good candidates for analysis using whole bone contours. Figure 4 compares BV/TV values from the cylindrical method and the whole bone method for these 2 bones. The BV/TV values for ET50 give nearly identical results, while the BV/TV for the whole bone of ET46 gives lower values than the cylindrical method. We attribute this to the large macropore that is clearly visible in the lower right corner of the ET46 image (Figure 1A). Exclusion of this large open area from the VOI would likely raise the BV/ $\mathrm{TV}$ to a value similar to the cylindrical method. When drawing the cylinders, we were careful to exclude this space from the contours we drew. Because similar trends are observed above with the whole bone contours as with the cylinders, we suggest that drawing cylindrical contours is a valid method of image analysis, and may give better contrast between well- and poorly preserved bones because large structural pores or cracks can more easily be excluded from the VOI. Thus, the appropriate microCT analysis method can be applied based upon the macrostructure of a given bone rather than concerns about accuracy of the results.

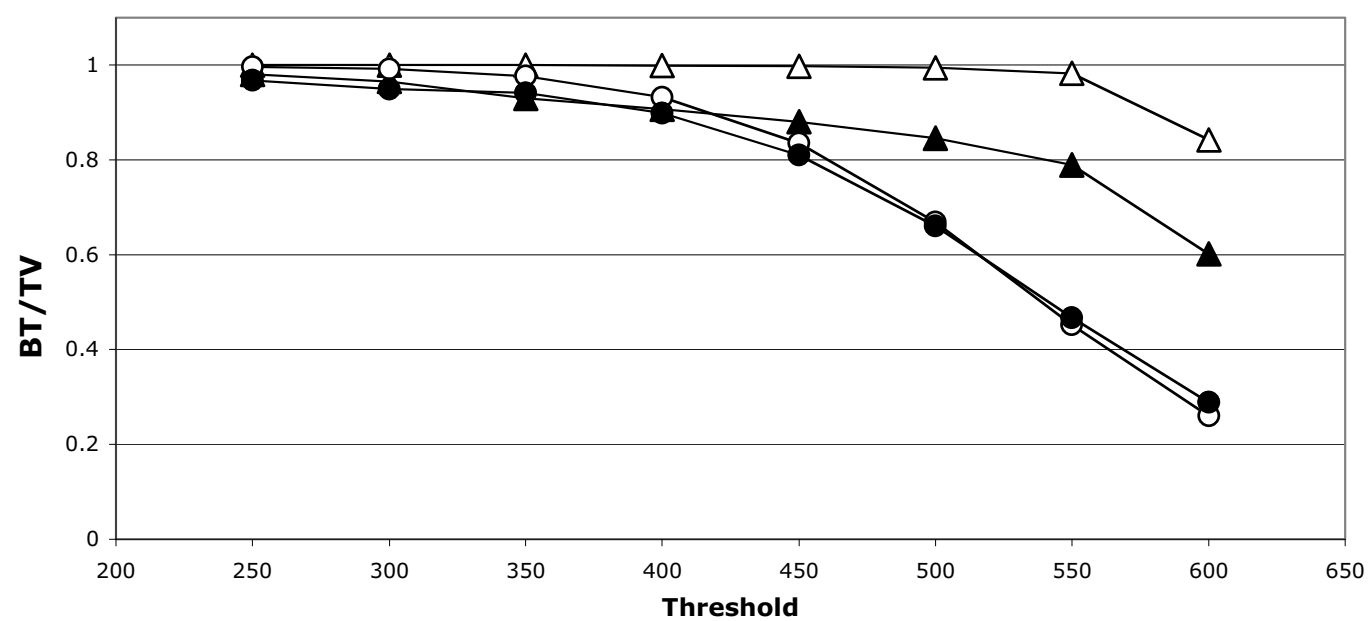

Figure 4 Comparison of cylindrical contour method with analysis of whole bone using low-resolution images. $\triangle=E T 46$, $\mathrm{O}=$ ET50. White points are analysis using cylindrical method, black points are whole bone contours. Details of VOI designation are explained in the text.

A faster analysis of the bones may be a simple measurement of BV/TV at a single threshold, and at a threshold of 500 the differences between the BV/TV of the bones appear to be greatest. Plotting bone volume fraction against collagen recovery (Figure 5) illustrates a trend in which bones with 
higher collagen recovery show a higher BV/TV. Our preliminary results suggest that a BV/TV below approximately 0.8 at this threshold, especially when coupled with a large measurement error, may indicate low collagen recovery. With so few data points, such conclusions are at best tentative, but we anticipate that with further work these trends will remain valid and that microCT may offer the opportunity to prescreen bones for collagen recovery.

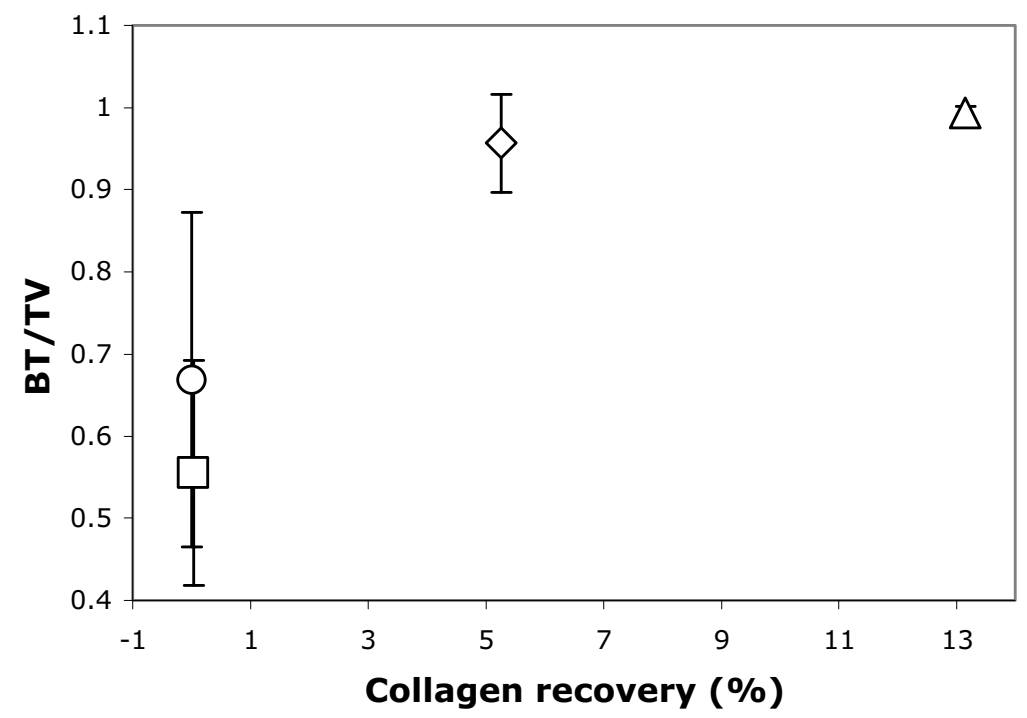

Figure 5 Average BV/TV for Etton bones analyzed using cylindrical contour method (threshold $=500$ ) plotted against collagen recovery. Error bars show 1 standard deviation $(n=4$ for all samples except ET12, where $n=3)$. $\diamond=\mathrm{ET} 12, \square=\mathrm{ET} 18, \triangle=\mathrm{ET} 46, \bigcirc=$ ET50.

\section{CONCLUSION}

We have presented results of microCT scans and analysis of 4 variably preserved bones from Etton, Cambridgeshire, UK. Our preliminary results indicate that bone volume fraction (BV/TV) measurements of low-resolution images can predict collagen recovery values. The BV/TV measurements can be done equally well by designating the whole bone, or several cylindrical volumes within the bone, as the volume of interest. This microCT method has the potential to be developed into a rapid, nondestructive prescreening method for collagen recovery for ${ }^{14} \mathrm{C}$ dating, and could also be a powerful technique for studying bone diagenesis more generally. Because the bone volume fraction measurements are spatially resolved, these results also offer the promise of determining the most suitable location within a bone to take a sample in order to maximize collagen recovery. We are currently in the process of scanning and analyzing more variably preserved bones from Etton, and will report the results of this larger project in due course.

\section{ACKNOWLEDGMENTS}

The authors gratefully recognize the National Science Foundation (Award \# 0722751) and the University of Scranton for funding, and Rashesh Kapadia of Scanco for helpful discussions about image analysis. We also thank Richard Sabin of the Natural History Museum for providing the bones and Dr Fiona Brock of ORAU for providing the collagen recovery and stable isotope data on the bones we analyzed. 


\section{REFERENCES}

Brock F, Higham T, Bronk Ramsey C. 2007. Radiocarbon dating bone samples recovered from gravel sites. Grant report, ORAU, Oxford.

Bronk Ramsey C, Higham T, Bowles A, Hedges R. 2004. Improvements to the pretreatment of bone at Oxford. Radiocarbon 46(1):155-63.

Brown AS, Leamen L, Cucevic V, Foster FS. 2005. Quantitation of hemodynamic function during developmental vascular regression in the mouse eye. Investigative Ophthalmology and Visual Science 46(7): 2231-7.

Collins MJ, Nielsen Marsh CM, Hiller J, Smith CI, Roberts JP, Prigodich RV, Wess TJ, Csapò J, Millard AR, Turner-Walker G. 2002. The survival of organic matter in bone: a review. Archaeometry 44(3):383-94.

Hedges REM. 2002. Bone diagenesis: an overview of processes. Archaeometry 44(3):319-28.

Hedges REM, Millard AR, Pike AWG. 1995. Measurements and relationships of diagenetic alteration of bone from three archaeological sites. Journal of Archaeological Science 22(2):201-9.

Huppertz A, Wildung D, Kemp BJ, Nentwig T, Asbach P, Rasche FM, Hamm B. 2009. Nondestructive insights into composition of the sculpture of Egyptian Queen Nefertiti with CT. Radiology 251:233-40.

Laib A, Barou O, Vico L, Lafage-Proust M-H, Alexandre C, Rüegsegger P. 2000. 3-D micro-computed tomography of trabecular and cortical bone architecture with application to a rat model of immobilization osteoporosis. Medical and Biological Engineering and Computing 38(3):326-32.

Lam YM, Chen XB, Marean CW, Frey CJ. 1998. Bone density and long bone representation in archaeological faunas: comparing results from $\mathrm{CT}$ and photon densitometry. Journal of Archaeological Science 25(6): $559-70$.

Müller R, Van Campenhout H, Van Damme B, Van der Perre G, Dequeker J, Hildebrand T, Rüegsegger P.
1998. Morphometric analysis of human bone biopsies: a quantitative structural comparison of histological sections and micro-computed tomography. Bone 23(1):59-66.

Murphy WA, zur Nedden D, Gostner P, Knapp R, Recheis W, Seidler H. 2003. The Iceman: discovery and imaging. Radiology 226:614-29.

Nielsen-Marsh CM, Hedges REM. 1999. Bone porosity and the use of mercury intrusion porosimetry in bone diagenesis studies. Archaeometry 41(1):165-74.

Nielsen-Marsh CM, Hedges REM. 2000. Patterns of diagenesis in bone I: the effects of site environments. Journal of Archaeological Science 27(12):1139-50.

Olejniczak AJ, Grine FE. 2006. Assessment of the accuracy of dental enamel thickness measurements using microfocal X-ray computed tomography. Anatomical Record A 288(3):263-75.

Parfitt AM, Drezner MK, Glorieux FH, Kanis JA, Malluche H, Meunier PJ, Ott SM, Recker RR. 1987. Bone histomorphometry: standardization of nomenclature, symbols, and units. Journal of Bone Mineral Research 2(6):595-610.

Peters OA, Laib A, Rüegsegger P, Barbakow F. 2000. Three-dimensional analysis of root canal geometry using high-resolution computed tomography. Journal of Dental Research 79(6):1405-9.

Rüegsegger P, Koller B, Müller R. 1996. A microtomographic system for the nondestructive evaluation of bone architecture. Calcified Tissue International 58(1):24-9.

Squire M, Brazin A, Keng Y, Judex S. 2008. Baseline bone morphometry and cellular activity modulate the degree of bone loss in the appendicular skeleton during disuse. Bone 42(2):341-9.

Turner-Walker G, Syversen U. 2002. Quantifying histological changes in archaeological bones using BSESEM image analysis. Archaeometry 44(3):461-8. 